\title{
OPEN Design of hybrid narrow-band plasmonic absorber based on chalcogenide phase change material in the infrared spectrum
}

\begin{abstract}
Israel Alves Oliveira ${ }^{1}$, Igor Leonardo Gomes de Souza ${ }^{2 \varpi}$ \& Vitaly Felix Rodriguez-Esquerre ${ }^{1}$
Structures absorbing electromagnetic waves in the infrared spectral region are important optical components in key areas such as biosensors, infrared images, thermal emitters, and special attention is required for reconfigurable devices. We propose a three-dimensional metal-dielectric plasmonic absorber with a layer of PCM's (Phase Change Materials). The phase shift effects of PCMs are numerically analyzed, and it is possible to obtain a shifting control of the resonant absorption peaks between the amorphous and crystalline states using the Lorentz-Lorenz relation. By using this empirical relation, we analyzed the peak absorption shift at intermediate phases between the amorphous and the crystalline. The geometric parameters of the structure with the PCM layer in the semi-crystalline state were adjusted to exhibit strong absorption for normal incidence. The effects of the oblique incidence on the absorption for the TM and TE polarization modes were also analyzed. Our results demonstrate that PCMs have great potential for reconfigurable nanophotonic devices.
\end{abstract}

Electromagnetic absorber structures based on metamaterials and plasmonics that are able to achieve almost perfect electromagnetic absorption, are very important devices because of their wide applications, such as

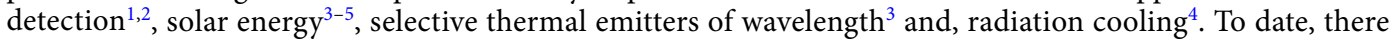
are several reports of absorbers operating over different bands of the electromagnetic spectrum. Most of them are based on a Metal-Dielectric-Metal configuration, having specific absorption bandwidths, restricting their high performance applications ${ }^{5-7}$. Recent studies demonstrated an extraordinary absorption response from the Bi-based MPA in ultra-broadband, polarization insensitive, and almost omnidirectional NIR (near infrared) perfect absorber by using Bi nanodiscs in an metal-isolator-metal configuration ${ }^{8}$. The GST chalcogenide phase change materials, (this includes GeTe), can be quickly ${ }^{9}$ and repeatedly ${ }^{10}$ switched between amorphous and crystalline states by appropriate thermal, electric, or optical stimuli ${ }^{11-13}$. Until now, GST chalcogenides have been regarded as strong candidates for realizing reconfigurable and nonvolatile all-optical devices ${ }^{14}$, consequently, several applications have been proposed, such as all-optical switching ${ }^{15}$, optical filters ${ }^{16}$, and reconfigurable metasurfaces ${ }^{17,18}$. Plasmonic absorbers based on PCMs including GeTe were analyzed in Refs. ${ }^{19-21}$, this resonant structures composed by metals and PCM's as insulators with sub-wavelength dimensions have been used as mirrors to suppress transmission by increasing the reflection and inducing destructive interference from reflected light at resonant wavelengths absorbed on the structure ${ }^{22}$. These proposed works explore the great contrast of the material's optical constants between its amorphous and crystalline phases in the regions of the electromagnetic spectrum from the visible (VIs) to the infrared (IR).

$\mathrm{VO}_{2}$ based PCM devices have been used earlier and they allow resonance tuning when combined with metallic structures. In this way, thermal reconfigurable absorbers have been also investigated. However, the metallic phase imposes a lower temperature limit of $68^{\circ} \mathrm{C}$ being considered a volatile material ${ }^{23,24}$. Non volatile PCM could be obtained when chalcogenides are used. Nowadays, one of the most used materials is the $\mathrm{Ge}_{2} \mathrm{Sb}_{2} \mathrm{Te}_{5}$ (GST 225). Plasmonic and hybrid metamaterial reconfigurable absorbers have been proposed using the GST225 ${ }^{25-27}$. Several chalcogenides have been used in the infrared region $\left(\mathrm{Ge}_{3} \mathrm{Sb}_{2} \mathrm{Te}_{6}, \mathrm{Ge}_{2} \mathrm{Sb}_{2} \mathrm{Te}_{4}, \mathrm{Ge}_{2} \mathrm{SbTe}_{4}, \mathrm{Ag}_{4} \mathrm{em}_{3} \mathrm{Sb}_{67} \mathrm{Te}_{26} \mathrm{e} \mathrm{GeTe}\right)$ They are deposited over polar substrates $\left(\mathrm{SiC}, \mathrm{Al}_{2} \mathrm{O}_{3}\right.$ e $\left.\mathrm{SiO}_{2}\right)$ exhibiting high refractive index contrast between the amorphous phase, where they are lossless and transparent, while the crystalline phase exhibits high attenuation. Consequently, the absorption occurs in the substrate for the amorphous phase while for the crystalline phase it

${ }^{1}$ Graduate School of Electrical Engineering, Federal University of Bahia, Salvador 40210-630, Brazil. ${ }^{2}$ Institute of Science, Technology and Innovation at the Federal University of Bahia (ICTI-UFBA), Camaçari 42802-721, Brazil. ${ }^{\varpi}$ email: ilgsouza@ufba.br 
happens in the PCM layer ${ }^{28}$. Also, PCMs based in $\mathrm{In}_{3} \mathrm{SbTe}_{2}$ (IST) which can switch from dielectric to metallic states are also designed to operate in the infrared region ${ }^{29}$.

GeTe has a crystallization temperature higher than the GST, exhibiting also different transition times which are needed for $\mathrm{NVM}^{30,31}$ applications an additional practical applications ${ }^{32,33}$. Although the above mentioned advantages of the GeTe, there are still few absorbers based on this material. Our results demonstrate the feasibility of using $\mathrm{GeTe}$ for the design of reconfigurable devices.

The main characteristic of PCMs is that they are not volatile at room temperature and can change gradually between phases (amorphous, crystalline or intermediate) in a periodic way with billions cycles per second (nanosecond or faster time response) by applying an external stimulus, which can be optical, temperature variation or electrical voltage $\mathrm{e}^{9,34}$. The Induced crystallization of $\mathrm{GeTe}$ can be done using laser pulses that give the material enough heat for the phase transition. In this type of stimulus, only a small section of the GeTe crystallizes, i.e. the area where the laser hit the material, limiting the number of possible applications. The transition from amorphous (a-GeTe) to crystalline (c-GeTe) phase in GeTe using conduction heating is completely reversible and happens after a heating of approximately $200^{\circ} \mathrm{C}$, in this phase change there is a difference of approximately six orders in the magnitude of its resistivity. This characteristic can be used for data storage devices ${ }^{35}$. The transition between phases can also be done using short and high intensity voltage pulses (joule heating), these pulses use DC sources, an advantage of this external stimulus is the fast switching between states approximately $10 \mathrm{~ns}$ from the a-GeTe state to $\mathrm{c}-\mathrm{GeTe}$, and $5 \mathrm{~ns}$ from the $\mathrm{c}-\mathrm{GeTe}$ state to a-GeTe ${ }^{36}$.

Although some works on GST-based absorbers have been published in the literature, the proposed one in this work demonstrates an alternative and ideal solution for designing reconfigurable absorbers based on GeTe PCM's with dielectric substrate. We propose a novel absorber for applications in optical communications with absorption above $95 \%$, with values of $98 \%$ in the second and third optical communications window covering the $\mathrm{O}+\mathrm{E}+\mathrm{S}+\mathrm{C}$ bands.

The proposed absorber is reconfigurable in the range spectrum of 1100-1900 nm. Using the effective medium theory, we explored the crystallization fractions of GeTe to tune the resonance peak of the absorber. We also evaluated the behavior of absorbers for oblique angles of incidence (up to $45^{\circ}$ ). Here we propose some possibilities to tune the resonant peak of this absorber: (1) with the crystallization fraction; (2) with the geometric parameters. In addition, we also evaluated the effects of the absorption with the variation of the geometrical parameters and the physical absorption mechanisms through the analysis of the spatial distribution of the electromagnetic fields and the current density inside the absorber.

\section{Structure's design and methods}

In this study, a hybrid, tunable and reconfigurable perfect absorber in the infrared spectral range considering a plasmonic metamaterial using a chalcogenide phase change material ( $\mathrm{GeTe}$ ) is proposed and theoretically investigated. Our nanoplasmonic structure consists of a periodic array placed directly on the surface of uniform silicon ( $\mathrm{Si}$ ). The building blocks of our design consist of square gold ( $\mathrm{Au}$ ) nanoantennas above a dielectric $\mathrm{Si}_{3} \mathrm{~N}_{4}$ spacer layer and a GeTe layer (Fig. 1a,b). In comparison with the planar absorber (Fig. 1c), the hybrid device with a phase change material can dramatically enhance the absorbance due to the induced electric and magnetic modes at the resonance. For the analysis of the absorber behavior and simulations, we will use the unit cell (Fig. 1d) which represents the geometric parameters of the structure. The Au square nanocubes have two geometric parameters, $\mathrm{h}_{\mathrm{Au}}$ and $\mathrm{w}_{\mathrm{Au}}$ with $150 \mathrm{~nm}$ and $160 \mathrm{~nm}$, respectively. The insulator spacer $\left(\mathrm{Si}_{3} \mathrm{~N}_{4}\right)$ and GeTe mirror thicknesses are $125 \mathrm{~nm}$ and $130 \mathrm{~nm}$, respectively. The whole structure resides on a silicon substrate with $145 \mathrm{~mm}$ thickness. The lattice constant in both $\mathrm{x}$ - and $\mathrm{y}$-directions is $\mathrm{p}=300 \mathrm{~nm}$. The relative permittivity of gold can be obtained through the Drude-Lorentz dispersion ${ }^{37}$. The refractive indexes of the insulators $\left(\mathrm{Si}_{3} \mathrm{~N}_{4}\right.$ and $\left.\mathrm{Si}\right)$ in the analyzed spectrum region are 2.0 and 3.5, respectively. The optical constants of GeTe are explained in Ref. ${ }^{12}$ and they are shown in Fig. 2a,b. The numerical method to calculate the absorption coefficient of the structure is the finite element method $(\mathrm{FEM})^{38}$, using the commercial software COMSOL ${ }^{39}$. The periodic boundary conditions are used for the lateral surfaces and PMLs (perfectly absorbing layers) are applied along the $z$ direction to eliminate undesired boundary reflections. Since the substrate film is thicker than the penetration depth of the incident light, it will block the propagation, consequently, the transmittance $(T)$ of the structure is almost zero, resulting in the absorption equation $A=1-R$, with $\mathrm{R}$ being the reflection). In particular, the fast phase transition in PCMs occurs gradually, and the switching speed is considerably high ${ }^{40}$ rather than suddenly (time responses of about nanoseconds and picoseconds), increasing the possibility of adjustments. During the phase transition, the PCM film can be assumed to be in the intermediate phase composed by different regions of amorphous and crystalline atoms and this phase change kinetics can be accomplished by heating ${ }^{41}$.

Based on the optical constants of the PCM in both phases and using the theory of the effective medium ${ }^{42}$, it is possible to obtain the permittivity at intermediate levels of crystallization for this material. This relation is called Lorentz-Lorentz, as follows:

$$
\frac{\varepsilon_{\text {eff }}(\lambda)-1}{\varepsilon_{\text {eff }}(\lambda)+2}=m \cdot \frac{\varepsilon_{\text {crystalline }}(\lambda)-1}{\varepsilon_{\text {crystalline }}(\lambda)+2}+(1-m) \cdot \frac{\varepsilon_{\text {amorphous }}(\lambda)-1}{\varepsilon_{\text {amorphous }}(\lambda)+2}
$$

where $\lambda$ is the operation wavelength, $\varepsilon_{\text {eff }}$ is the permittivity of the GeTe at the crystallization level $m$, and, $\varepsilon_{\text {crystalline }}$ and, $\varepsilon_{\text {amorphous }}$ are permittivity of GeTe in fully crystallized and fully amorphous states, respectively. We use the relations:

$$
\varepsilon(\lambda)=n^{2}(\lambda)-k^{2}(\lambda)
$$




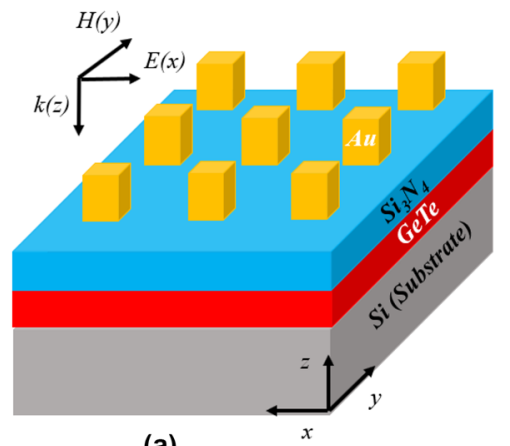

(a)

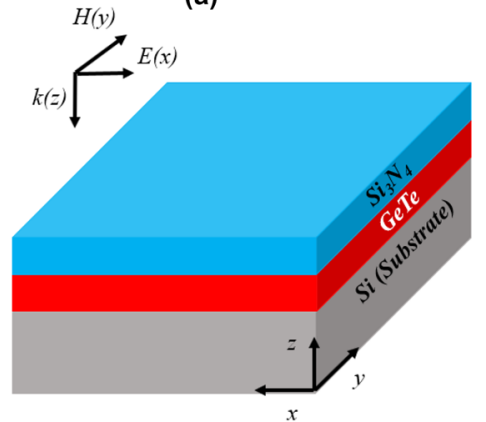

(c)

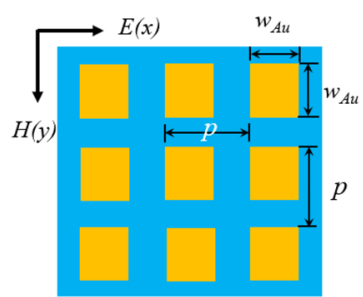

(b)

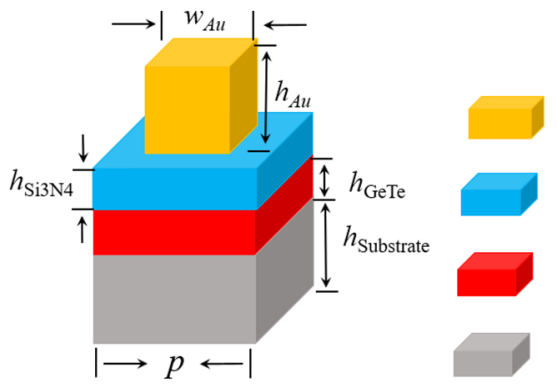

$A u$

$\mathrm{Si}_{3} \mathrm{~N}_{4}$

GeTe

Si (Substrate)

(d)

Figure 1. (a) Schematic representation of the hybrid absorber; (b) top view of the absorber the hybrid plasmonic absorber; (c) representation of the planar control absorber without Au nanoantennas; (d) unit cell of the hybrid plasmonic absorber.
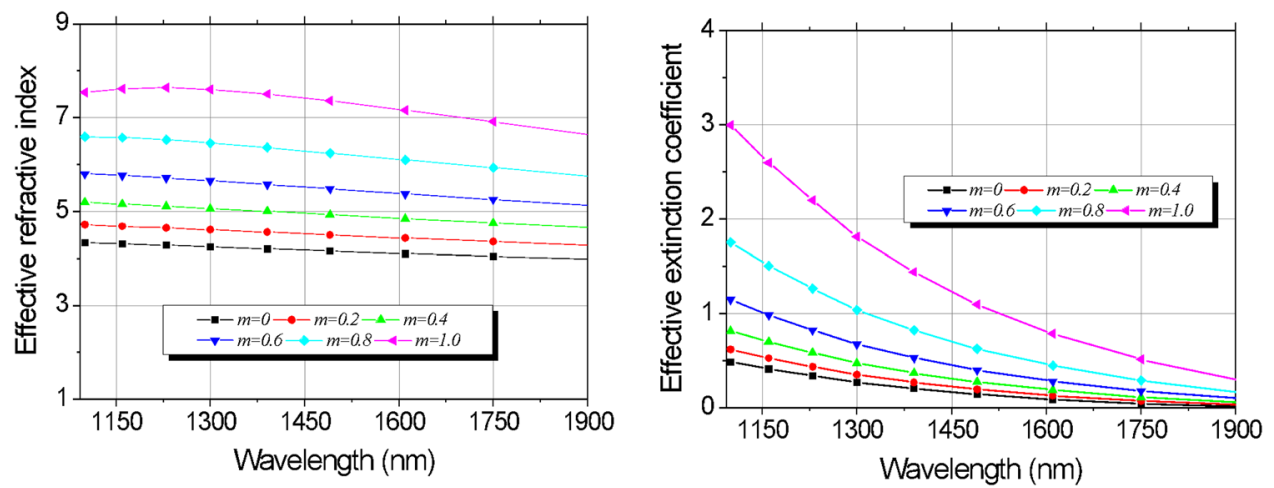

Figure 2. (a) Refractive index and; (b) extinction coefficient of the GeTe using Eq. (1).

$$
\tilde{\varepsilon}(\lambda)=-2 n k
$$

In order to calculate the real and imaginary parts of the GeTe permittivity, next, we could determine the $\varepsilon_{\text {eff }}(\lambda)$ for each fraction of crystallization by using Eq. (1). The effective refractive index and extinction coefficient are shown in Fig. 2a,b, respectively, they depend on the wavelength and on the levels of crystallization $m$.

\section{Results and discussions}

With the structure properly designed, we compared the results with a continuous planar control device, where the golden layers are absent (see Fig. la,c). These results can be seen in Fig. 3a,b. The proposed structure exhibits high absorption in both phases (amorphous and crystalline) at different wavelength peaks (1279 nm and $1664 \mathrm{~nm}$, respectively), while the planar structure (without golden squares) presents a sharp drop in absorption when the length of wave shifts to the right and in the crystalline state, the absorption decreases in the intermediate range of the analyzed spectrum, and again reaches $90 \%$ absorption at the wavelength near $1900 \mathrm{~nm}$. Structures based on planar absorbers are ideal for Fabry Perot resonators ${ }^{19,43,44}$, where the conditions of maintaining resonance within the cavity are present. For the proposed structure in this article, cavity resonances can be obtained by using periodic array geometry. 
(a)

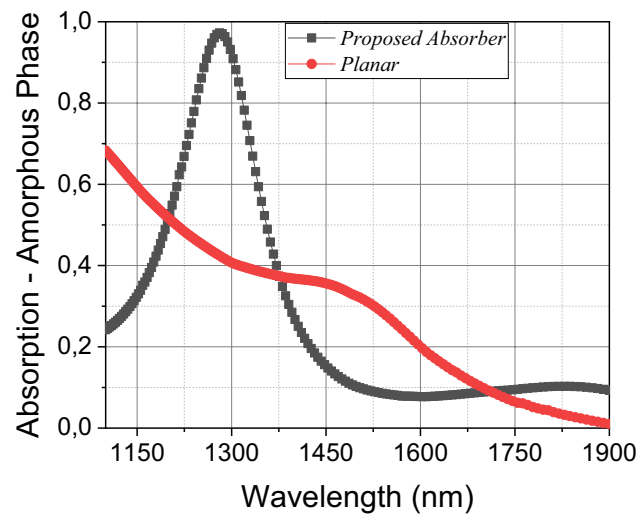

(b)

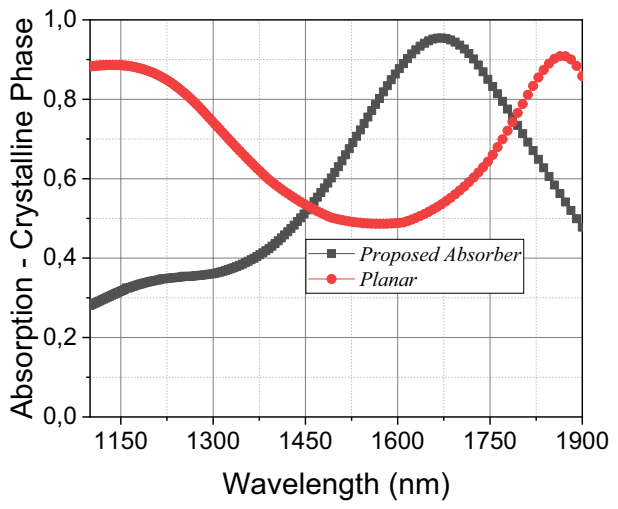

Figure 3. Absorption spectrum of the proposed absorber compared to the planar metadevice on phases: (a) amorphous and; (b) crystalline.

(a)

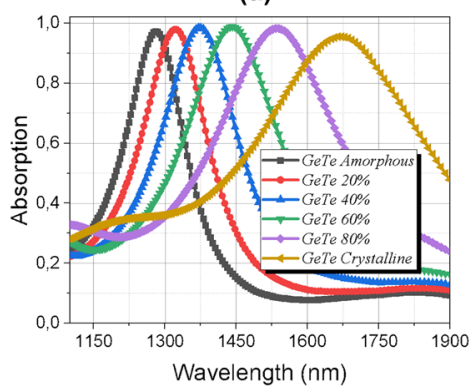

(b)

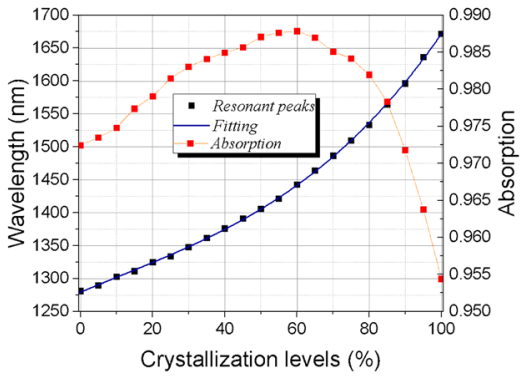

(c)

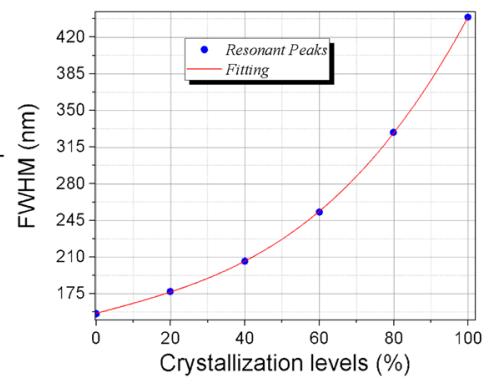

Figure 4. (a) The evolution of the absorption spectrum for different crystallization levels (b) by a fit polynomial of degree 3. The percentages shown in the legend correspond to the GeTe crystallization levels (c) Variation of the Full Width Half Maximum (FWHM) as a function of the crystallization of GeTe, by a fit also polynomial of degree 3.

The Fig. 4a shows the evolution of the absorption spectrum as PCM crystallization level increases for normal incidence. The infrared region of the spectrum is sensitive to phase changes due to the strong electronic polarizability of GeTe in the crystalline state, producing a high contrast in its refractive index ${ }^{45}$. We also numerically analyzed the behavior of the resonant peak position and value with the crystallization fraction (Fig. 4b). The red curve shows the absorption coefficient for each peak, it is possible to analyze that the structure has an absorption above $95.5 \%$ for all resonant peaks. The blue curve shows that the first peak is shifted to the right when the crystallization fraction of GeTe increases. Using high precision statistical methods, we predict a function to obtain the resonance peak as a function of the crystallization fraction of $\mathrm{GeTe}$, as follow:

$$
\lambda\left(m_{\%}\right)=1279.15+2.32 m-7.87 \cdot 10^{-2} m^{2}+2.38 \cdot 10^{-4} m^{3}
$$

where, $m$ is the crystallization fraction of GeTe. This equation can be used to modulate any resonance peak as a function of $m$ in that interval of 1100-1900 nm.

The results shown in Fig. 4a revealed an increasing in the FWHM, along the range of crystallization of $\mathrm{GeTe}$. The peak gets wider because the extinction coefficient $(\mathrm{k})$ of gold varies almost linearly as the wavelength increases, resulting in absorption in a larger region of wavelengths, consequently, the FWHM increases. Analogously to obtaining the behavior of this parameter, this evolution can be expressed by the equation as follows:

$$
\text { FWHM }\left(m_{\%}\right)=156.15+9.5 \cdot 10^{-1} \mathrm{~m}-3.97 \cdot 10^{-8} \mathrm{~m}^{2}+1.89 \cdot 10^{-4} \mathrm{~m}^{3}
$$

where, $m_{\%}$ is the crystallization fraction of GeTe. This equation can be used to analyze the FWHM for any fraction of crystallization of GeTe. Figure 4c shows the behavior of the FWHM.

The absorption responses as a function of the incident angle and crystallization phases of the PCM have been also analyzed. For TE polarization, the resonant wavelength remains constant up to $30^{\circ}$, then it shifts slightly to shorter wavelengths, as shown in Fig. $5 \mathrm{a}-\mathrm{f}$. The numerical results show absorption close to the unit for all analyzed phases for normal incidence $\left(\theta=0^{\circ}\right)$ and oblique angles of incidence up to $45^{\circ}$.

The absorption performances for TM polarization are shown in Fig. 6a-f. The numerical results show high absorption for all presented angles (from $0^{\circ}$ to $45^{\circ}$ ). It is possible to observe a great angular insensitivity, which 
(a)

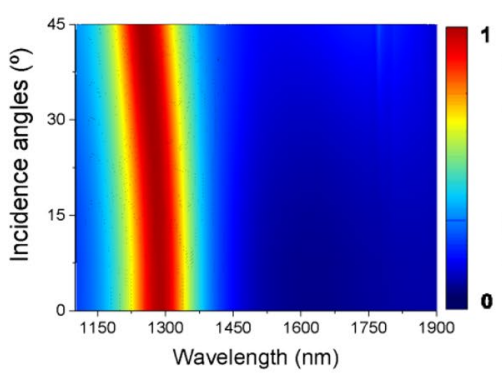

(d)

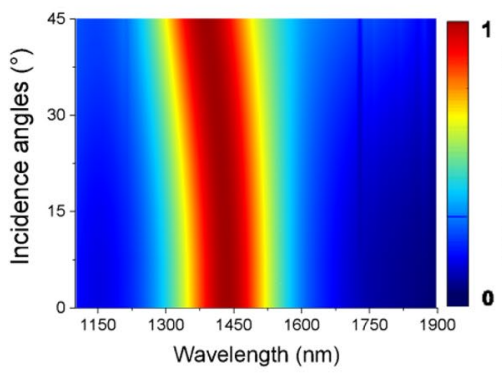

(b)

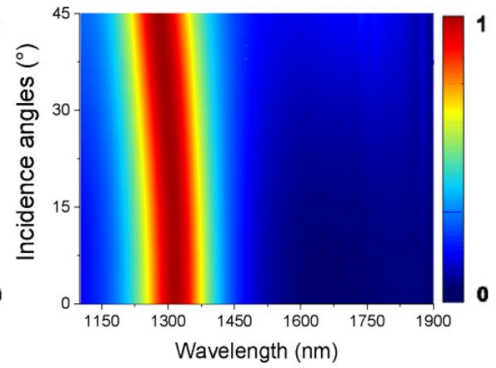

(e)

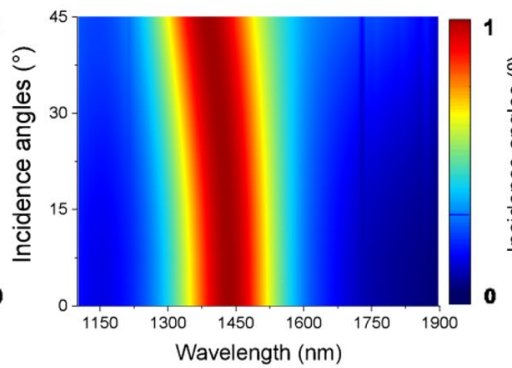

(c)

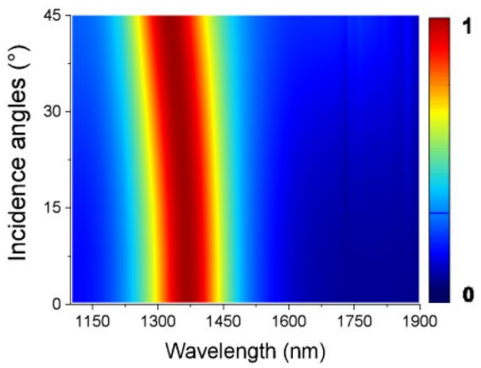

(f)

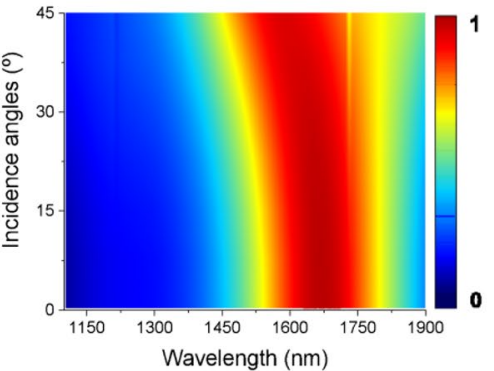

Figure 5. Dependence of the absorption for incident angles from $0^{\circ}$ to $45^{\circ}$ in TE polarization in the $1100-$ $1900 \mathrm{~nm}$ spectrum on: (a) amorphous phase $(m=0 \%),(\mathbf{b}) m=20 \%,(\mathbf{c}) m=40 \%$, (d) $m=60 \%,(\mathbf{e}) m=80 \%$, and (f) crystalline phase $(m=100 \%)$.

(a)

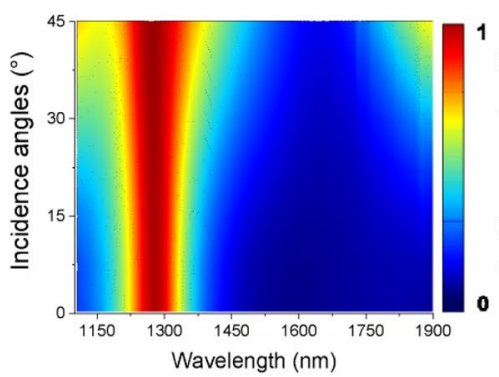

(d)

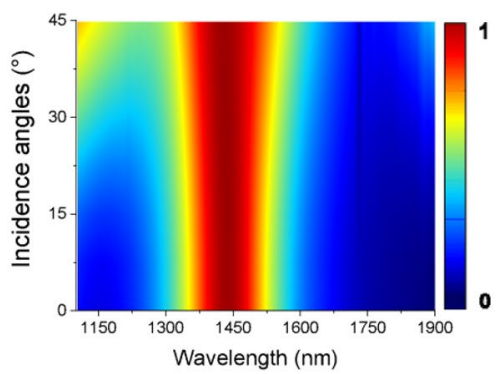

(b)

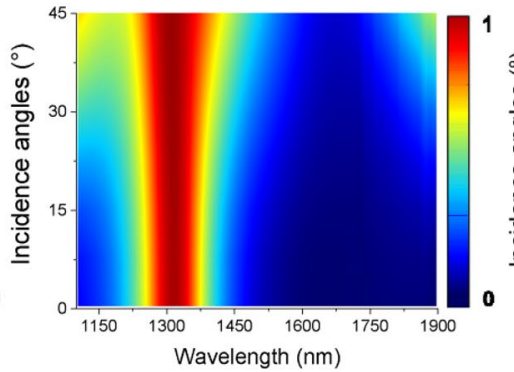

(e)

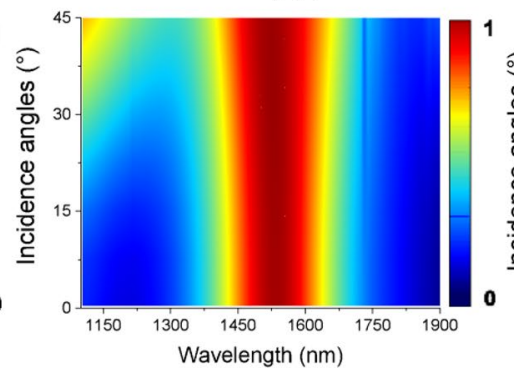

(c)

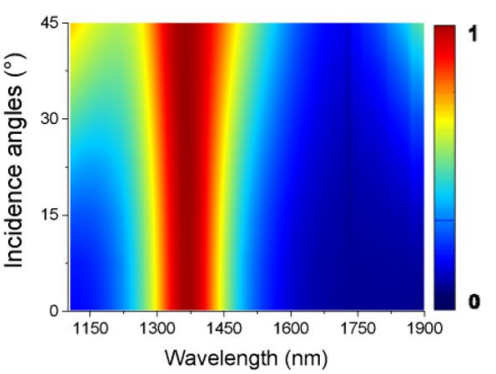

(f)

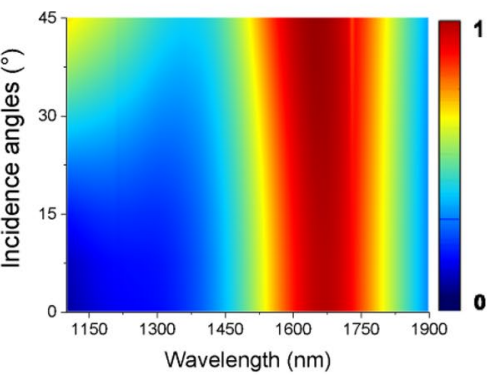

Figure 6. Dependence of the absorption for incident angles from $0^{\circ}$ to $45^{\circ}$ in TM polarization in the $1100-$ $1900 \mathrm{~nm}$ spectrum on: (a) amorphous phase $(m=0 \%),(\mathbf{b}) m=20 \%,(\mathbf{c}) m=40 \%,(\mathbf{d}) m=60 \%,(\mathbf{e}) m=80 \%$, and (f) crystalline phase $(m=100 \%)$.

is ideal for several practical applications ${ }^{46-49}$. The results also show that there is a high absorption at wavelengths close to the resonant peak, which increases as the incident angle increases. Thus, the proposed hybrid absorber behaves as wideangle insensitive polarization device across the infrared spectral region. The wideangle behavior of the proposed device can be explained by the subwavelength size of the nanoantenna which can act as a broadband impedance-matched structure ${ }^{50}$. The unitary cell of the proposed absorber is about $300 \mathrm{~nm} \times 300 \mathrm{~nm} \times 400 \mathrm{~nm}$ which is critical to achieve wideangle absorption. Numerical simulations ${ }^{51}$ have found that the $\mathrm{A}(\theta)$ rapidly drops to small values when the unitary cell is large, in this way the incident angle of the 
(a)

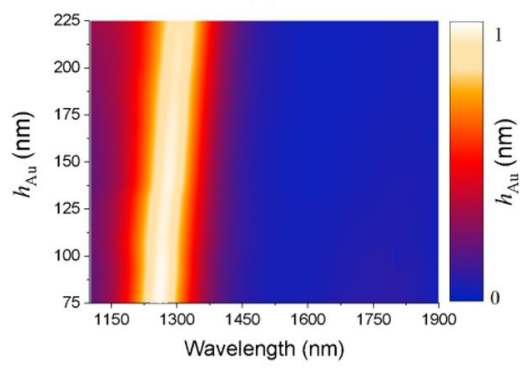

(b)

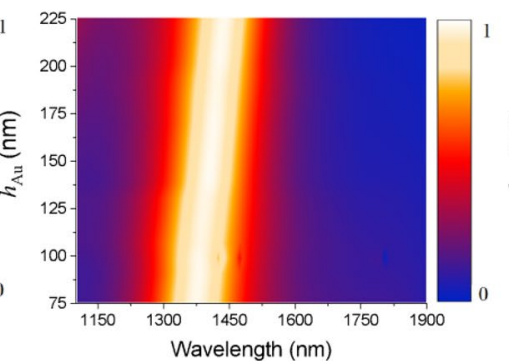

(c)

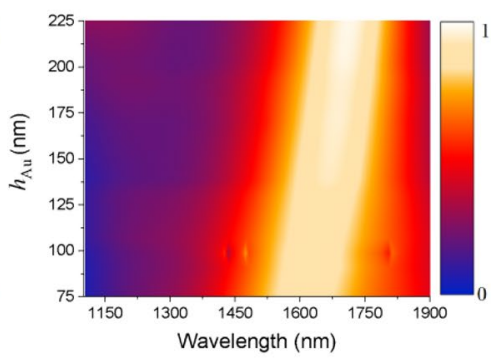

Figure 7. Absorption map in relation to the thickness of golden square $\left(h_{\text {Au }}\right)$ scanning for $(\mathbf{a})$ amorphous; $(\mathbf{b})$ semi-crystalline and; (c) crystalline phases.

$\mathbf{E}$

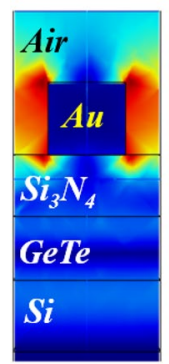

(a)
$\mathbf{J}$

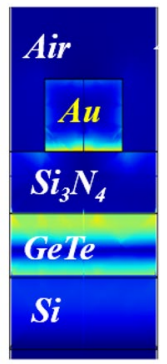

(b)
$\mathbf{H}$

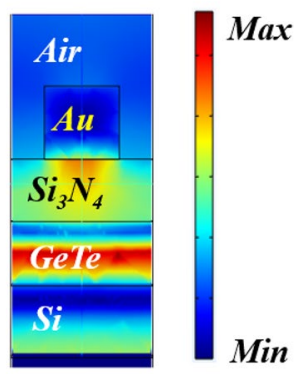

(c)

Figure 8. Spatial distribution of (a) normalized electric field; (b) normalized current density and; (c) normalized magnetic field of the absorber on resonant wavelength at $1550 \mathrm{~nm}$ and $m=82.5 \%$.

electromagnetic radiation has low influence on the response of the absorber. Moreover, because only a unitary cell has been considered in the vertical direction with subwavelength thickness, a wideangle operation could be expected since narrow band absorbance will occur when several layers are stacked.

To analyze the manufacturing tolerance of the device, we studied the absorption effects of the thickness of the golden squares $\left(h_{\mathrm{Au}}\right)$. A sweep has been performed for widths between 75 and $225 \mathrm{~nm}$, which are smaller and larger than the ideal values $(150 \mathrm{~nm})$. The results can be seen in Fig. 7a-c. and they demonstrate that the absorption remains high and the peak displacement is proportional to the variation in the thickness of the $h_{\mathrm{Au}}$ metallic layer. Furthermore, the peak shifting and the increasing of the FHWM caused by crystallization variation do not interfere with the absorption efficiency, even for considerably large or small thicknesses.

In order to explain the physical mechanism of the absorber, we present the spatial distribution of the fields $\mathrm{E}, \mathrm{H}$, and the current density $\mathrm{J}$ in the $\mathrm{xz}$ plane at the resonant wavelength of $1550 \mathrm{~nm}$, with a crystallization fraction of $\mathrm{m}=82.5 \%$ (Fig. $8 \mathrm{a}-\mathrm{c}$ ). The results show an electric field strength between the side spacing of the top metallic layer. Current density is distributed between the semiconductor and the bottom side of the metallic layer. The intense magnetic field present in the PCM layer favors the interaction between the silicon nitride dielectric layer and the metallic layer, causing interference and generating SPP's, due to the semiconductor characteristic so that the absorption remains high. This constructive interference was caused by the choice of the physical and geometrical parameters of the structure for phase compensation, resulting in total absorption at the resonant peak. Spatial distribution of the absorption reveals that performance is higher at the interface between metal-dielectric ${ }^{52}$.

Likewise, the spatial distributions of the fields of the normalized $\mathbf{E}$, J and $\mathbf{H}$, respectively, for the same wavelength $(\lambda=1550 \mathrm{~nm})$, but with the PCM phase in an amorphous state and the results can be analyzed in Fig. $9 \mathrm{a}-\mathrm{c}$, therefore, non-resonant wavelength. The results reveal that there is no field weakening at the metal-air interface. Because of the GeTe's magnetic field increases, the resonance is reflected at this point and shifted to shorter wavelengths ${ }^{53}$, according to the fitting Eq. (4). Current density is almost completely eliminated, concentrating on specific points on the upper layer.

The effects of the width of the metallic layer of this absorber $\left(w_{\mathrm{Au}}\right)$ were also analyzed for values in the interval $150-170 \mathrm{~nm}$. Its peak resonance capability can be adjusted by shifting GeTe phases according to Fig. 10a-e. The results showed that there is a displacement of the resonance peak for longer wavelengths due to the increase in the layer width in all phases of GeTe. The increasing in the width of the gold nanoantenna results in a large area, since the current density is concentrated in this region (Fig. 8b) the absorption peak value increases.

Additionally, and based on methods of multiple linear regression, the curves were fitted to determine a function in which the fraction of crystallization of GeTe and the thickness of the metallic layer are independent variables and can be adjusted by the equation: 


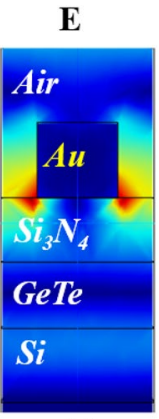

(a)
$\mathbf{J}$

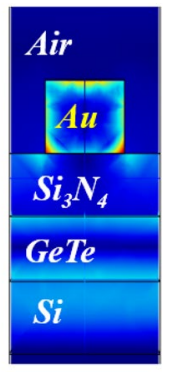

(b)
$\mathbf{H}$

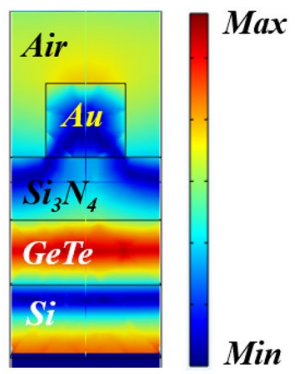

(c)

Figure 9. Spatial distribution of (a) normalized electric field; (b) normalized current density and; (c) normalized magnetic field of the absorber on resonant wavelength at $1550 \mathrm{~nm}$ and $m=0 \%$.

(a)

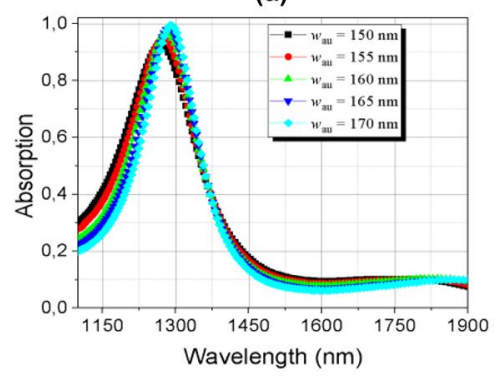

(d)

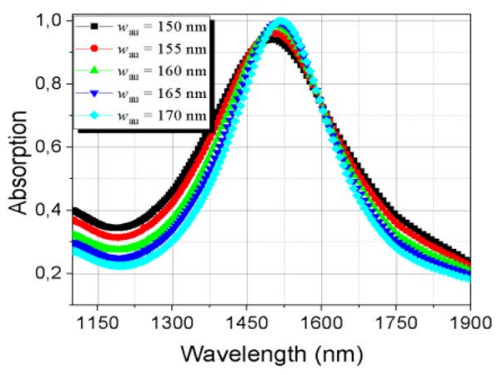

(b)

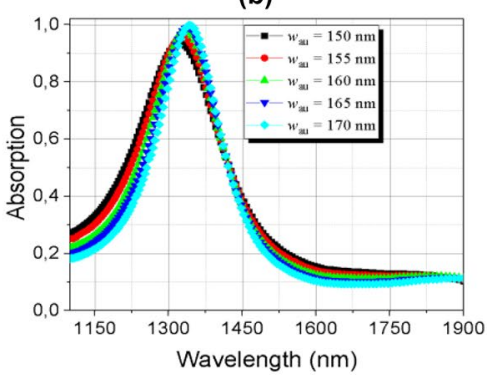

(e)

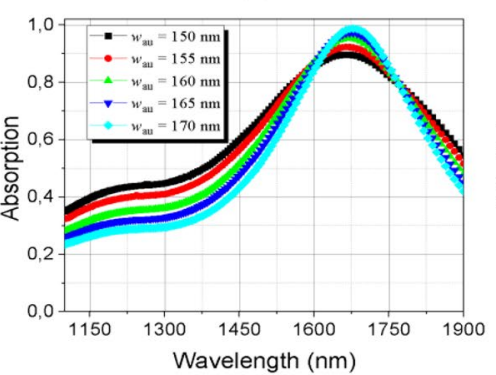

(c)

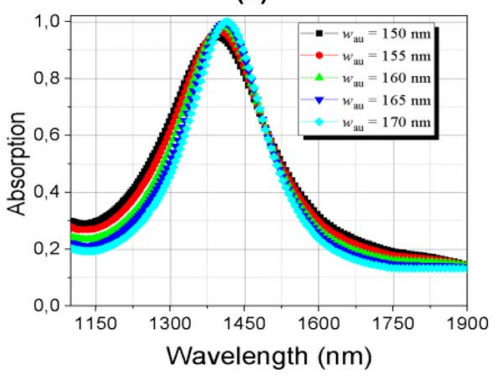

(f)

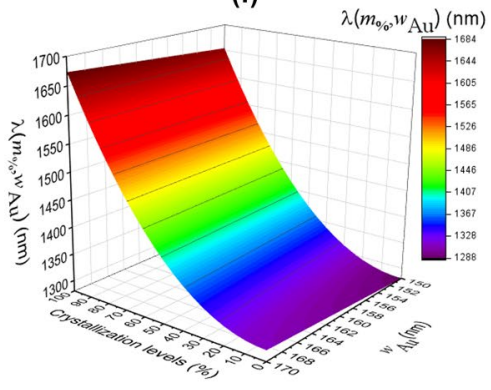

Figure 10. Absorption dependence of the $w_{\mathrm{Au}}$ metallic layer width in the range of $1100-1900 \mathrm{~nm}(\mathbf{a})$ amorphous; (b) semi-crystalline $(\mathrm{m}=25 \%)$; (c) semi-crystalline $(\mathrm{m}=50 \%)$; $(\mathbf{d})$ semi-crystalline $(\mathrm{m}=75 \%) ;(\mathbf{e})$ crystalline phases and; (f) surface graph of the multivariable function.

$$
\lambda\left(m_{\%}, w_{A u}\right)=374.10^{-2} m^{2}+976.10^{-2} w_{A u}+1142,98
$$

here, $m_{\%}$ is the crystallization fraction of GeTe and wAu the thickness of gold. For the analysis of the behavior of resonance peaks during evolution (phase change), a surface graph is presented in Fig. 10f, where these two independent variables have been combined.

The absorption effects have been also analyzed by varying the thickness of the $h_{\mathrm{Si3N} 4}$ dielectric layer and their results are shown in Fig. 11a-e. The results show insensitivity to the variation of this geometric parameter. The variation of the thickness of the $\mathrm{Si}_{3} \mathrm{~N}_{4}$ almost does not interfere with the absorption response because the highest current density is located in the gold nanoantenna base and in the PCM (GeTe) layer. For this reason there was no need for linearization. Despite this, absorption remained high for all phases of PCM.

The effects of absorption by varying the layer thickness of the material with phase change have been also investigated and the results are presented in Fig. 12a-e. The results show that increasing the PCM layer results in a shift of the resonant peak to higher wavelengths, due to the change in material thickness and optical modulation caused by crystallization. The results also reveal that these displacements are slightly spaced. The curves were linearized as can be seen in Fig. 12-f, making it possible to reach linear functions to adjust resonant wavelengths with the thickness of the PCM and the linear expressions are presented in Table 1.

The effects on the absorption by varying the layer thickness of the silicon dielectric substrate material were also analyzed and the results are shown in Fig. 13a-e. They reveal that its variation interferes with absorption and that there is also a displacement of the resonant peaks. The curves were also linearly fitted, allowing the control of the peaks through linear functions shown in Table 2. The results of the linear dependence of the resonant 
(a)

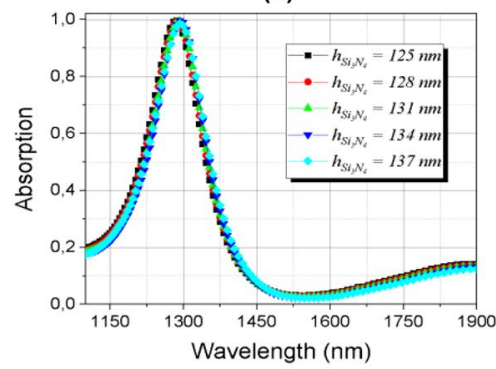

(d)

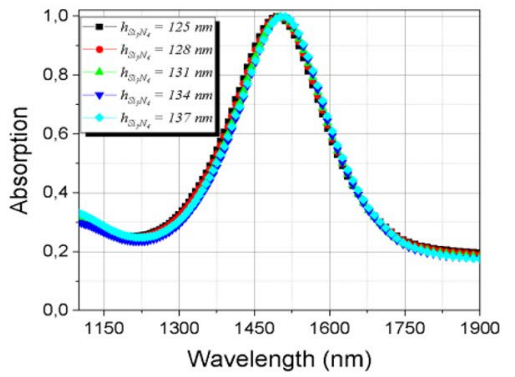

(b)

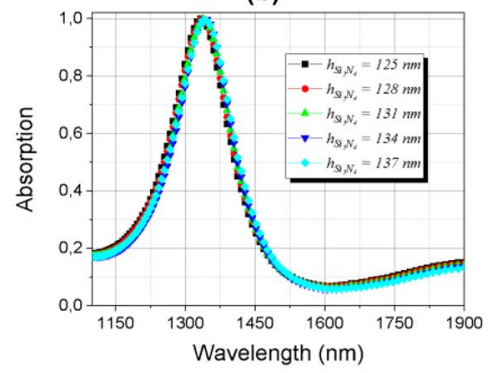

(c)

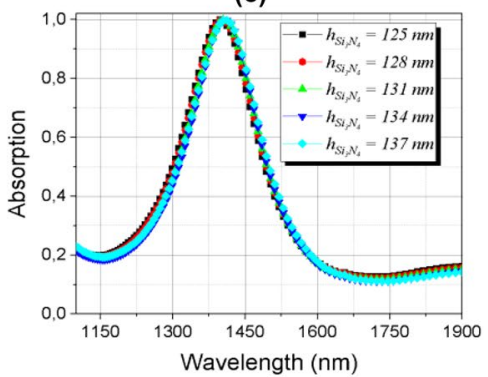

(e)

Figure 11. Absorption dependence of the dielectric thickness of $\mathrm{Si}_{3} \mathrm{~N}_{4} h_{\mathrm{Si} 3 \mathrm{~N} 4}$ in the range of $1100-1900 \mathrm{~nm}(\mathbf{a})$ amorphous; (b) semi-crystalline $(\mathrm{m}=25 \%)$; (c) semi-crystalline $(\mathrm{m}=50 \%)$; (d) semi-crystalline $(\mathrm{m}=75 \%) ;(\mathbf{e})$ crystalline phases.

(a)

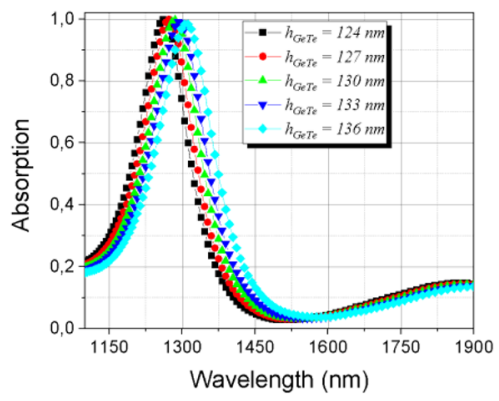

(d)

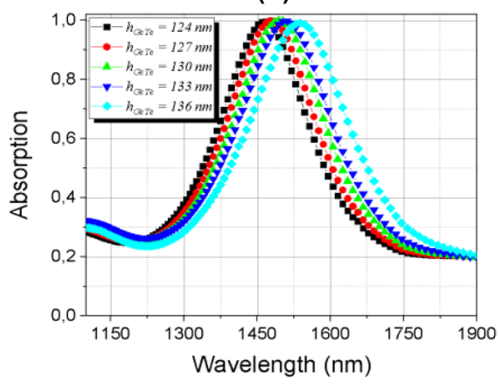

(b)

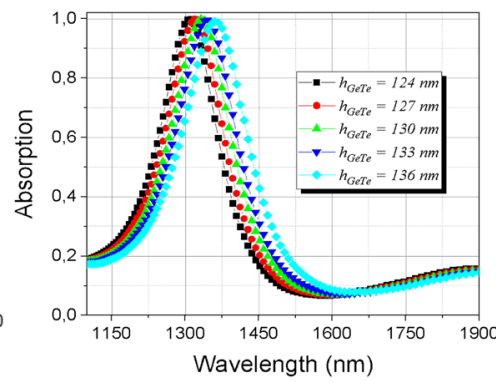

(e)

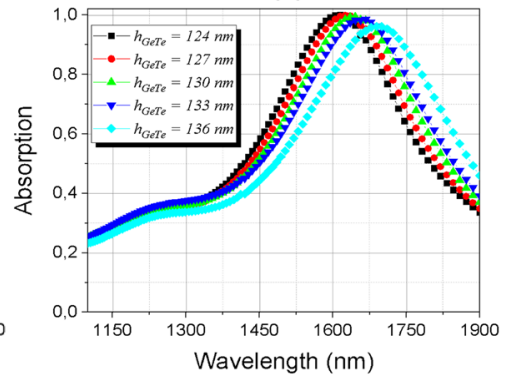

(c)

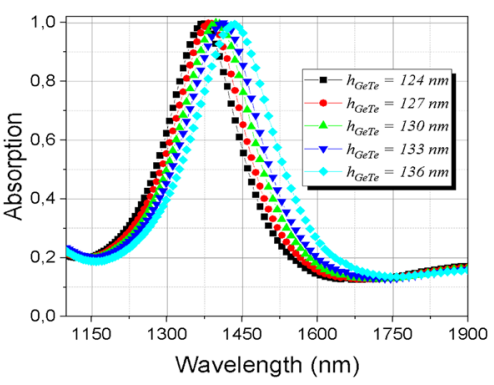

(f)

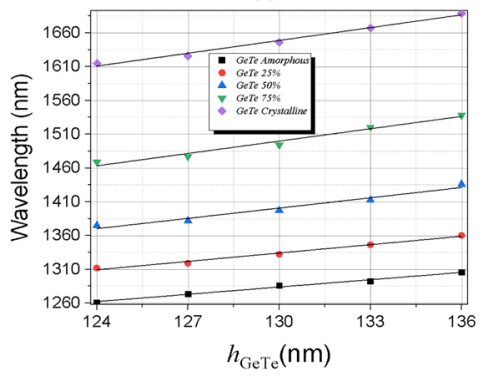

Figure 12. Absorption dependence of the PCM thickness $h_{\mathrm{GeTe}}$ in the range of $1100-1900 \mathrm{~nm}$ (a) amorphous; (b) semi-crystalline $(\mathrm{m}=25 \%)$; (c) semi-crystalline $(\mathrm{m}=50 \%)$; (d) semi-crystalline $(\mathrm{m}=75 \%)$; (e) crystalline phases and (f) linear dependence of the peaks of $(\mathbf{a}-\mathbf{e})$ with the thickness of the GeTe layer $\left(h_{\mathrm{GeTe}}\right)$.

peaks with the variation of the substrate layer thickness can be seen in Fig. 13-f. The thickness of the GeTe and silicon layers are related with the resonant cavity size, then, the resonance wavelength shift linearly to higher wavelength values when they increases.

The dependence of the absorption as a function of the periodicity of the structure is also analyzed as can be seen in Fig. 14a-e. The results showed that the increase in periodicity results in a shifting of the resonant peak to shorter wavelengths, indicating a linear decrease, shown in Fig. 14-f. Based on the presented results, the curves 


\begin{tabular}{|l|l|l|}
\hline GeTe's phase change & Linear expression & \\
\hline Amorphous $(\mathrm{m}=0 \%)$ & $\lambda\left(h_{\mathrm{GeTe}}\right)=3.61 h_{\mathrm{GeTe}}+814.79$ & $(7)$ \\
\hline SemiCrystalline $(\mathrm{m}=25 \%)$ & $\lambda\left(h_{\mathrm{GeTe}}\right)=4.14 h_{\mathrm{GeTe}}+796.30$ & $(8)$ \\
\hline SemiCrystalline $(\mathrm{m}=50 \%)$ & $\lambda\left(h_{\mathrm{GeTe}}\right)=5.08 h_{\mathrm{GeTe}}+740.45$ & $(9)$ \\
\hline SemiCrystalline $(\mathrm{m}=75 \%)$ & $\lambda\left(h_{\mathrm{GeTe}}\right)=6.11 h_{\mathrm{GeTe}}+705.91$ & $(10)$ \\
\hline Crystalline $(\mathrm{m}=100 \%)$ & $\lambda\left(h_{\mathrm{GeTe}}\right)=6.32 h_{\mathrm{GeTe}}+827.05$ & $(11)$ \\
\hline
\end{tabular}

Table 1. Linearization of the absorber resonant peaks with $h_{\mathrm{GeTe}}$.

(a)

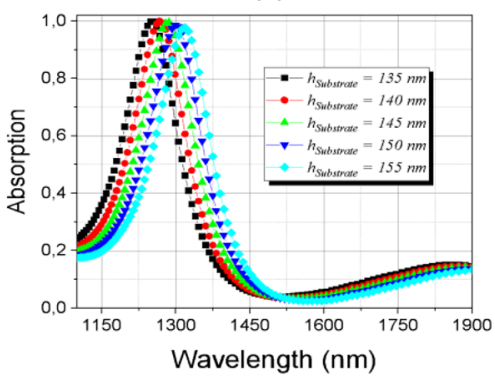

(d)

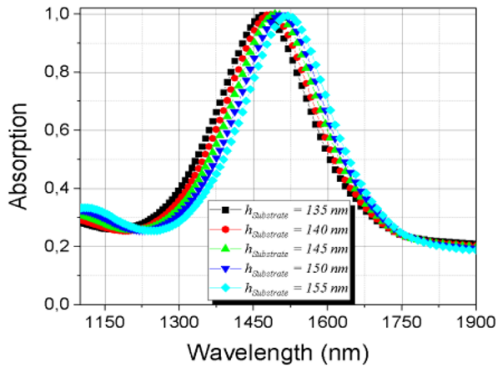

(b)

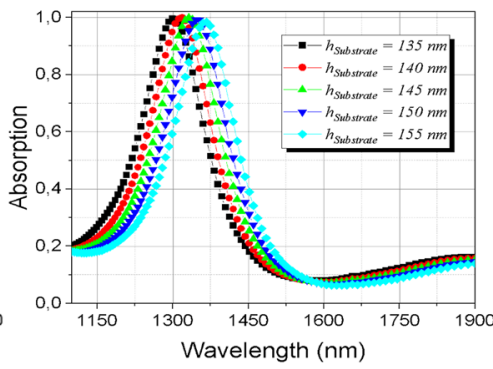

(e)

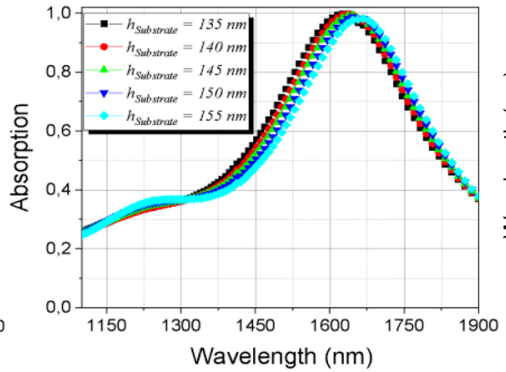

(c)

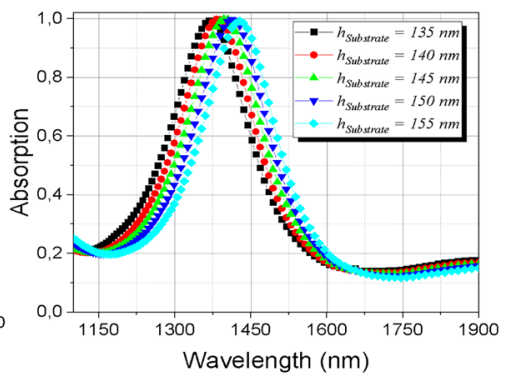

(f)

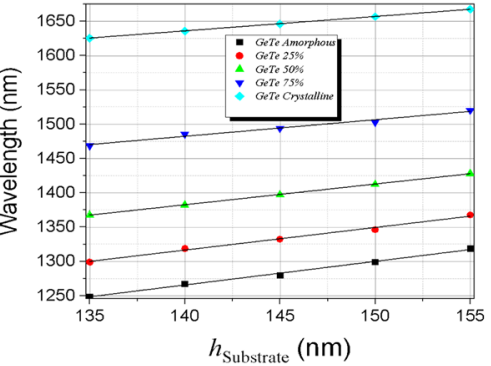

Figure 13. Absorption dependence of the silicon substrate thickness $h_{\text {Substrate }}$ in the range of 1100 to $1900 \mathrm{~nm}$ (a) amorphous; (b) semi-crystalline $(\mathrm{m}=25 \%)$; (c) semi-crystalline $(\mathrm{m}=50 \%)$; (d) semi-crystalline $(\mathrm{m}=75 \%)$; (e) crystalline phases and (f) linear dependence of the peaks of (a-e) with the thickness of the substrate thickness $\left(h_{\text {Substrate }}\right)$.

\begin{tabular}{|l|l|l|}
\hline GeTe's phase change & Linear expression \\
\hline Amorphous $(\mathrm{m}=0 \%)$ & $\lambda\left(h_{\text {Substrate }}\right)=3.30 h_{\text {Substrate }}+854.78$ & $(12)$ \\
\hline SemiCrystalline $(\mathrm{m}=25 \%)$ & $\lambda\left(h_{\text {Substrate }}\right)=3.30 h_{\text {Substrate }}+854.78$ & $(13)$ \\
\hline SemiCrystalline $(\mathrm{m}=50 \%)$ & $\lambda\left(h_{\text {Substrate }}\right)=3.02 h_{\text {Substrate }}+959.54$ & $(14)$ \\
\hline SemiCrystalline $(\mathrm{m}=75 \%)$ & $\lambda\left(h_{\text {Substrate }}\right)=2.42 h_{\text {Substrate }}+1143.70$ & $(15)$ \\
\hline Crystalline $(\mathrm{m}=100 \%)$ & $\lambda\left(h_{\text {Substrate }}\right)=2.10 h_{\text {Substrate }}+1342.35$ & $(16)$ \\
\hline
\end{tabular}

Table 2. Linearization of the absorber resonant peaks with $h_{\text {Substrate }}$

were fitted, making it possible to control the resonance peaks in a function of the variation in the periodicity of the absorber, which can be analyzed in Table 3.

\section{Conclusion}

In conclusion, we demonstrated a hybrid and multilayer three-dimensional resonant absorber, composed of chalcogenide phase change material (GeTe). The absorbers have been numerically analyzed and high optical modulation caused by the variation of the GeTe crystallization phase has been demonstrated. We numerically determined that the resonant peak wavelength can be controlled as a function of the crystallization fraction, as well as the physical parameters such as the thickness of the layers, periodicity and width of the upper gold layer. The resonant wavelength can be empirically obtained through linear, 3rd degree polynomials and multivariables expressions. The dependence of the structure on the incident angle up to $45^{\circ}$ was also analyzed and the absorption remained high regardless of polarization. The excellent results remain constant even for variations in the thickness of the gold layer to account for possible errors in the manufacturing process. The physics mechanism 
(a)

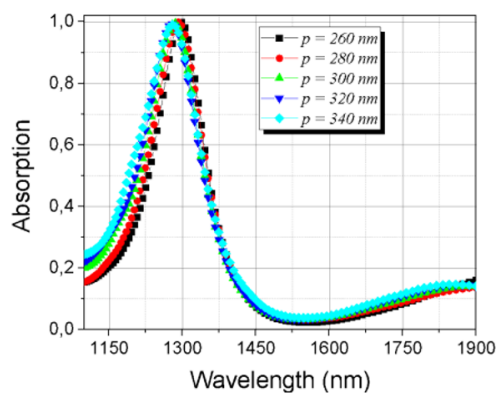

(d)

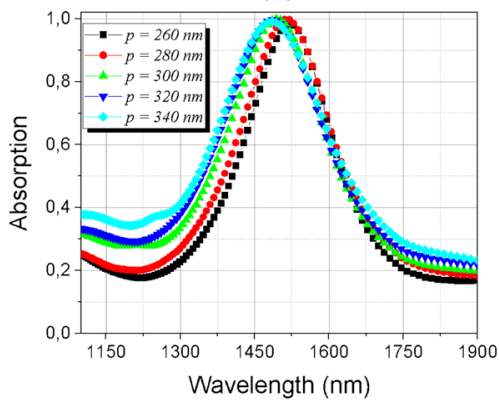

(b)

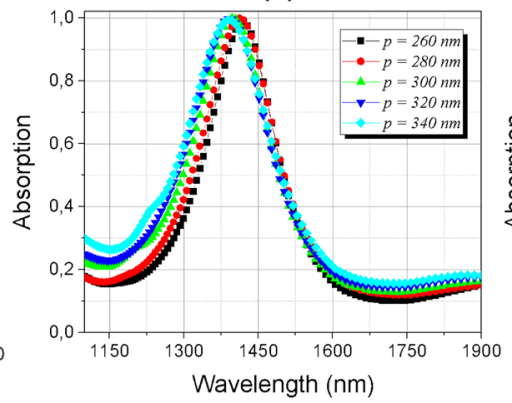

(e)

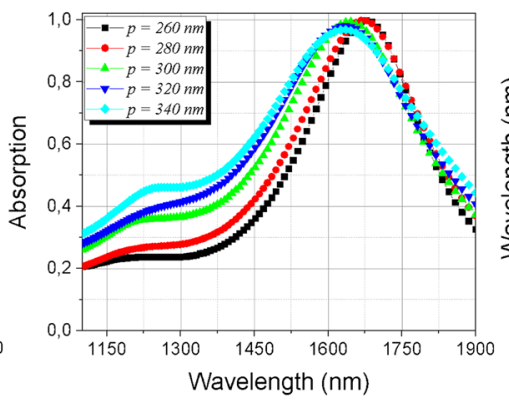

(c)

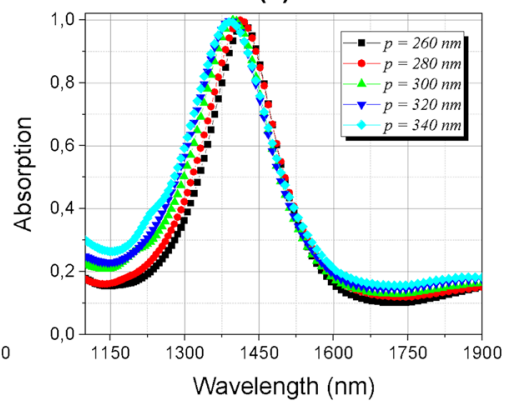

(f)

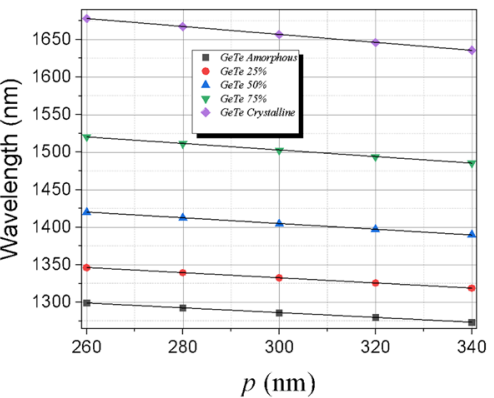

Figure 14. Absorption dependence of the periodicity $p$ in the range of 1100 to $1900 \mathrm{~nm}$ (a) amorphous; (b) semi-crystalline $(\mathrm{m}=25 \%)$; (c) semi-crystalline $(\mathrm{m}=50 \%)$; (d) semi-crystalline $(\mathrm{m}=75 \%)$; (e) crystalline phases and (f) linear dependence of the peaks of $(\mathbf{a}-\mathbf{e})$ with the thickness of the periodicity.

\begin{tabular}{|l|l|l|}
\hline GeTe's phase change & Linear expression & \\
\hline Amorphous $(\mathrm{m}=0 \%)$ & $\lambda(p)=-0.32 p+1381.93$ & $(17)$ \\
\hline SemiCrystalline $(\mathrm{m}=25 \%)$ & $\lambda(p)=-0.34 p+1435.35$ & $(18)$ \\
\hline SemiCrystalline $(\mathrm{m}=50 \%)$ & $\lambda(p)=-0.38 p+1519.21$ & $(19)$ \\
\hline SemiCrystalline $(\mathrm{m}=75 \%)$ & $\lambda(p)=-0.43 p+1633.67$ & $(20)$ \\
\hline Crystalline $(\mathrm{m}=100 \%)$ & $\lambda(p)=-0.53 p+1815.92$ & $(21)$ \\
\hline
\end{tabular}

Table 3. Linearization of the absorber resonant peaks with the periodicity $p$.

of the absorption and the phase change of the chalcogenide allows it to be used as a semiconductor and insulator, and this reconfigurability modify the concentration of the electromagnetic fields, displacing the structure's resonance peaks to others regions. We would consider the use of approaches based on neuromorphic systems in future proposals ${ }^{54}$. This structure can be an alternative solution for absorbers with potential applications for various technologies employed in reconfigurable nanophotonic devices.

Received: 6 August 2021; Accepted: 26 October 2021

Published online: 09 November 2021

\section{References}

1. Ji, Y. et al. High-performance metamaterial sensors based on strong coupling between surface plasmon polaritons and magnetic plasmon resonances. Results Phys. 14, 102397 (2019).

2. Cen, C. et al. High quality factor, high sensitivity metamaterial graphene-Perfect absorber based on critical coupling theory and impedance matching. Nanomaterials 10, 95 (2020).

3. Sergeant, N. P., Pincon, O., Agrawal, M. \& Peumans, P. Design of wide-angle solar-selective absorbers using aperiodic metaldielectric stacks. Opt. Express 17, 22800 (2009).

4. Li, J. et al. Broadband solar energy absorber based on monolayer molybdenum disulfide using tungsten elliptical arrays. Mater. Today Energy. 16, 100390 (2020).

5. Li, J. et al. Tunable broadband solar energy absorber based on monolayer transition metal dichalcogenides materials using $\mathrm{Au}$ nanocubes. Nanomaterials 10, 257 (2020).

6. Cai, L. et al. Simultaneous single-peak and narrowband thermal emission enabled by hybrid metal-polar dielectric structures. Appl. Phys. Lett. 115, 093505 (2019).

7. Wu, D. et al. The design of ultra-broadband selective near-perfect absorber based on photonic structures to achieve near-ideal daytime radiative cooling. Mater. Des. 139, 104-111 (2018).

8. Ozbay, I., Ghobadi, A., Butun, B. \& Turhan-Sayan, G. Bismuth plasmonics for extraordinary light absorption in deep sub-wavelength geometries. Opt. Lett. 45, 686 (2020).

9. Loke, D. et al. Breaking the speed limits of phase-change memory. Science 336, 1566-1569 (2012). 
10. Zhang, S. et al. Tunable narrowband shortwave-infrared absorber made of a nanodisk-based metasurface and a phase-change material $\mathrm{Ge}_{2} \mathrm{Sb}_{2} \mathrm{Te}_{5}$ layer. Appl. Opt. 59, 6309 (2020).

11. Zhu, H. et al. Tunable narrowband mid-infrared thermal emitter with a bilayer cavity enhanced Tamm plasmon. Opt. Lett. 43, 5230 (2018).

12. Wuttig, M., Bhaskaran, H. \& Taubner, T. Phase-change materials for non-volatile photonic applications. Nat. Photon 11, 465-476 (2017).

13. Chu, C. H. et al. Laser-induced phase transitions of $\mathrm{Ge}_{2} \mathrm{Sb}_{2} \mathrm{Te}_{5}$ thin films used in optical and electronic data storage and in thermal lithography. Opt. Express 18, 18383 (2010).

14. Zhang, Y. et al. Broadband transparent optical phase change materials for high-performance nonvolatile photonics. Nat. Commun. 10, 4279 (2019).

15. Zhang, Z. et al. All-optical switch and logic gates based on hybrid silicon- $\mathrm{Ge}_{2} \mathrm{Sb}_{2} \mathrm{Te}_{5}$ metasurfaces. Appl. Opt. 58, 7392 (2019).

16. Zhou, K. et al. Multichannel tunable narrowband mid-infrared optical filter based on phase-change material $\mathrm{Ge}_{2} \mathrm{Sb}_{2} \mathrm{Te}_{5} \mathrm{defect}$ layers. Appl. Opt. 59, 595 (2020).

17. Wang, Q. et al. Optically reconfigurable metasurfaces and photonic devices based on phase change materials. Nat. Photon 10, 60-65 (2015).

18. Chen, Y. G. et al. Hybrid phase-change plasmonic crystals for active tuning of lattice resonances. Opt. Express 21, 13691 (2013).

19. Gomes de Souza, I. L., Rodriguez-Esquerre, V. F. \& Alves Oliveira, I. Design of planar reconfigurable, tunable, and wide angle resonant absorbers for applications in the IR spectrum. Adv. Theory Simul. 4, 2100002 (2021).

20. Radi, Y., Simovski, C. R. \& Tretyakov, S. A. Thin perfect absorbers for electromagnetic waves: theory, design, and realizations. Phys. Rev. Appl. 3, 037001 (2015).

21. Liu, N., Mesch, M., Weiss, T., Hentschel, M. \& Giessen, H. Infrared perfect absorber and its application as plasmonic sensor. Nano Lett. 10, 2342-2348 (2010).

22. Woo, B. H. et al. Angle-dependent optical perfect absorption and enhanced photoluminescence in excitonic thin films. Opt. Express 25, 28619 (2017)

23. Kocer, H. et al. Thermal tuning of infrared resonant absorbers based on hybrid gold $-\mathrm{VO}_{2}$ nanostructures. Appl. Phys. Lett. 106, $161104(2015)$.

24. Kats, M. A. et al. Vanadiu dioxide as a natural disordered metamaterial: Perfect thermal emission and large broadband negative differential thermal emittance. Phys. Rev. X. 3, 041004 (2013)

25. Ruiz de Galarreta, C. et al. Reconfigurable multilevel control of hybrid all-dielectric phase-change metasurfaces. Optica. 7, 476 (2020).

26. Abdollahramezani, S. et al. Dynamic hybrid metasurfaces. Nano Lett. 21, 1238-1245 (2021).

27. Tian, X. et al. Wavelength-selective, tunable and switchable plasmonic perfect absorbers based on phase change materials $\mathrm{Ge}_{2} \mathrm{Sb}_{2} \mathrm{Te}_{5}$. EPL (Europhys. Lett.) 128, 67001 (2020).

28. Heßler, A., Bente, I., Wuttig, M. \& Taubner, T. Ultra-thin switchable absorbers based on Lossy phase-change materials. Adv. Opt. Mater. 9, 2101118 (2021).

29. Heßler, A. et al. $\mathrm{In}_{3} \mathrm{SbTe}_{2}$ as a programmable nanophotonics material platform for the infrared. Nat. Commun. 12, 924 (2021)

30. Eliseev, N. N. et al. Wide range optical and electrical contrast modulation by laser-induced phase transitions in GeTe thin films. Results Phys. 19, 103466 (2020).

31. Bathaei, N., Weng, B. \& Sigmarsson, H. Growth study of GeTe phase change material using pulsed electron-beam deposition. Mater. Sci. Semicond. Process. 96, 73-77 (2019).

32. Chen, M., Rubin, K. A. \& Barton, R. W. Compound materials for reversible, phase-change optical data storage. Appl. Phys. Lett. 49, 502-504 (1986).

33. Chen, Y. et al. Unraveling the crystallization kinetics of supercooled liquid GeTe by ultrafast calorimetry. Cryst. Growth Des. 17, 3687-3693 (2017).

34. Jeong, Y., Bahk, Y. \& Kim, D. Dynamic terahertz plasmonics enabled by phase-change materials. Adv. Optical Mater. 8, 1900548 (2019).

35. Gwin, A. H., Kodama, C. H., Laurvick, T. V., Coutu, R. A. Jr. \& Taday, P. F. Improved terahertz modulation using germanium telluride (GeTe) chalcogenide thin films. Appl. Phys. Lett. 107, 031904 (2015).

36. Gill, M., Lowrey, T. \& Park, J. Ovonic unified memory-A high-performance nonvolatile memory technology for stand-alone memory and embedded applications. in 2002 IEEE International Solid-State Circuits Conference. Digest of Technical Papers (Cat. No.02CH37315) (IEEE). https://doi.org/10.1109/isscc.2002.993006.

37. Rakić, A. D., Djurišić, A. B., Elazar, J. M. \& Majewski, M. L. Optical properties of metallic films for vertical-cavity optoelectronic devices. Appl. Opt. 37, 5271 (1998).

38. Rubio-Mercedes, C. E. \& Hernández-Figueroa, H. E. Padé Boundary Conditions for the Finite-Element Modeling of Arbitrary Planar Junctions. J. Light Technol. 22, 669 (2004)

39. COMSOL. http://www.comsol.com. Accessed 01 July 2021.

40. Michel, A. U. et al. Advanced optical programming of individual meta-atoms beyond the effective medium approach. Adv. Mater. 31, 1901033 (2019).

41. Wang, J., Wang, L. \& Liu, J. Overview of phase-change materials based photonic devices. IEEE Access 8, 121211-121245 (2020).

42. Aspnes, D. E. Local-field effects and effective-medium theory: A microscopic perspective. Am. J. Phys. 50, 704-709 (1982).

43. Gomes de Souza, I. L. \& Rodriguez-Esquerre, V. F. Design of planar and wideangle resonant color absorbers for applications in the visible spectrum. Sci. Rep. 9, 7045 (2019).

44. de Souza, I. L. G. \& Rodriguez-Esquerre, V. F. Wavelength-selective near unity absorber based on Fabry-Pérot nanoresonators. J. Microw. Optoelectron. Electromagn. Appl. 20, 219-227 (2021).

45. Huang, B. \& Robertson, J. Bonding origin of optical contrast in phase-change memory materials. Phys. Rev. B. 81, 081204(R) (2010).

46. Raty, J. et al. A quantum-mechanical map for bonding and properties in solids. Adv. Mater. 31, 1806280 (2018).

47. Gomes de Souza, I. L., Rodriguez-Esquerre, V. F. \& Rêgo, D. F. Wide-angle filters based on nanoresonators for the visible spectrum. Appl. Opt. 57, 6755 (2018).

48. Shin, H., Yanik, M. F., Fan, S., Zia, R. \& Brongersma, M. L. Omnidirectional resonance in a metal-dielectric-metal geometry. Appl. Phys. Lett. 84, 4421-4423 (2004).

49. Kocer, H., Butun, S., Li, Z. \& Aydin, K. Reduced near-infrared absorption using ultra-thin lossy metals in Fabry-Perot cavities. Sci. Rep. 5, 8157 (2015).

50. Avitzour, Y., Urzhumov, Y. A. \& Shvets, G. Wide-angle infrared absorber based on a negative-index plasmonic metamaterial. Phys. Rev. B. 79, 045131 (2009).

51. Landy, N. I., Sajuyigbe, S., Mock, J. J., Smith, D. R. \& Padilla, W. J. Perfect metamaterial absorber. Phys. Rev. Lett. 100, 207402 (2008).

52. Chen, J. et al. Polarization-independent almost-perfect absorber controlled from narrowband to broadband. Opt. Express 25, 13916 (2017).

53. Jeong, H.-D. \& Lee, S.-Y. Tunable plasmonic absorber using a nanoslit array patterned on a $\mathrm{Ge}_{2} \mathrm{Sb}_{2} \mathrm{Te}_{5}$-inserted Fabry-Pérot resonator. J. Lightw. Technol. 36, 5857-5862 (2018). 
54. Wu, C. et al. Programmable phase-change metasurfaces on waveguides for multimode photonic convolutional neural network. Nat. Commun. 12, 96 (2021).

\section{Acknowledgements}

The authors would like to thank CNPQ (Process: 309100/2018-6), FAPESP/MCTI/MC Process: 2015/24517-8, CAPES, UFBA, ICTI-UFBA, FAPESB (079/2016) and CAPES PRINT-Projeto Cidades Inteligentes.

\section{Author contributions}

All the authors conceived and planned the simulations, wrote and revised the Manuscript, I.A.O. performed the numerical simulations and prepared the figures, All the authors performed the physical behavior analyses and analysed the data, I.A.O., I.L.G.S. and V.F.R.E. revised all the numerical simulations. All authors provided critical feedback and helped shape the research, analysis and manuscript.

\section{Competing interests}

The authors declare no competing interests.

\section{Additional information}

Correspondence and requests for materials should be addressed to I.L.G.d.

Reprints and permissions information is available at www.nature.com/reprints.

Publisher's note Springer Nature remains neutral with regard to jurisdictional claims in published maps and institutional affiliations.

(c) (i) Open Access This article is licensed under a Creative Commons Attribution 4.0 International License, which permits use, sharing, adaptation, distribution and reproduction in any medium or format, as long as you give appropriate credit to the original author(s) and the source, provide a link to the Creative Commons licence, and indicate if changes were made. The images or other third party material in this article are included in the article's Creative Commons licence, unless indicated otherwise in a credit line to the material. If material is not included in the article's Creative Commons licence and your intended use is not permitted by statutory regulation or exceeds the permitted use, you will need to obtain permission directly from the copyright holder. To view a copy of this licence, visit http://creativecommons.org/licenses/by/4.0/.

(C) The Author(s) 2021 